\title{
Line Tension Effect upon Static Wetting
}

\author{
P. Seppecher ${ }^{1}$ \\ 1 Université de Toulon et du Var, BP 132, 83957 La Garde Cedex - France \\ e-mail: seppecher@univ-tln.fr
}

\begin{abstract}
Résumé - Effet de la tension de ligne sur le mouillage statique — La prise en compte d'une densité constante d'énergie le long de la ligne de contact conduit à des problèmes d'équilibre mal posés. Dans ce cas, l'énergie minimisée par les configurations d'équilibre est une énergie « relaxée » qui nécessite la prise en considération de phases de surface (i.e. de films infinitésimaux) sur les parois du récipient. Cette formulation permet de décrire les modifications de la loi de Young et donc d'équilibre entraînées par la présence de la tension de ligne.
\end{abstract}

Mots-clés : tension de ligne, mouillage statique, relaxation.

Abstract — Line Tension Effect upon Static Wetting — Adding simply, in the classical capillary model, a constant line density of energy along the contact line leads to ill-posed equilibrium problems. Then, when line tension is present, the equilibrium configuration minimizes a different energy: the "relaxed" energy, which explicitly depends on the presence of surface phases (i.e. infinitesimal films) on the boundary of the container. This formulation enables us to describe the modifications of the Young's law and then of equilibrium configurations which are due to line tension.

Keywords: line tension, static wetting, relaxation.

\section{INTRODUCTION}

In the simplest model for capillarity, one consider two phases $A$ and $B$ lying in a rigid container $\Omega$. One, at least, of the phases is incompressible and a constant surface energy $\gamma_{A B}$ is concentrated on the interface $S_{A B}$ which divides the two phases. The wetting properties of the wall $\partial \Omega$ of the

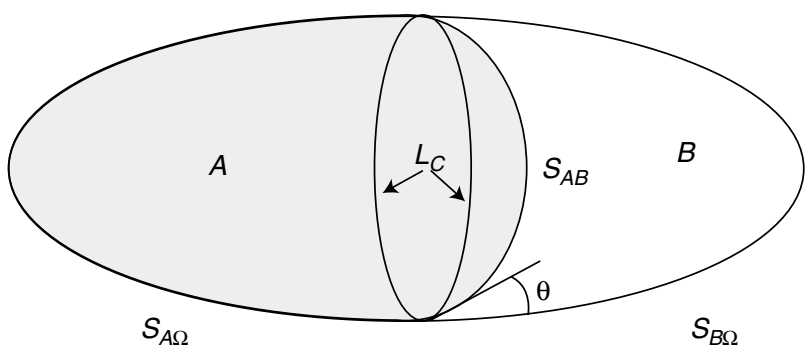

Figure 1

Notations. container are taken into account by considering constant surface energies $\gamma_{A \Omega}$ and $\gamma_{B \Omega}$ concentrated on the contact surfaces $S_{A \Omega}, S_{B \Omega}$ of the phases $A$ and $B$ on the wall. The contact line $L_{C}$, defined as the intersection of the interface and the wall of the container, plays an important role for describing equilibrium conditions. The associated contact angle $\theta$ is defined as the angle made by the interface and the wall, more precisely made by the normal vector of the interface $S_{A B}$ external with respect to $A$ and the normal vector of the wall $\partial \Omega$ external with respect to $\Omega$ (Fig. 1).

The equilibrium state is determined by the position of one of the phases, say $A$, and is given by the minimization of the capillary energy:

$$
E(A):=\gamma_{A B}\left|S_{A B}\right|+\gamma_{A \Omega}\left|S_{A \Omega}\right|+\gamma_{B \Omega}\left|S_{B \Omega}\right|
$$

where $\left|S_{A B}\right|,\left|S_{A \Omega}\right|,\left|S_{B \Omega}\right|$ denote the areas of the different interfaces. The total volume $|A|$ of the phase $A$ is fixed in this minimization procedure.

An interesting extension of this model is obtained by considering the possibility of a concentration of energy along 
the contact line [1-4]. Denoting $c$ the (constant) line tension, the equilibrium state is given by the minimization of the energy:

$$
F(A):=\gamma_{A B}\left|S_{A B}\right|+\gamma_{A \Omega}\left|S_{A \Omega}\right|+\gamma_{B \Omega}\left|S_{B \Omega}\right|+c\left|L_{C}\right|
$$

where | $L_{C} \mid$ denotes the length of the line $L_{C}$.

What are the equilibrium conditions in that case? And before all, is this minimization problem a well-posed problem? These are the questions we will discuss in the sequel. We will show that the problem is, in general, illposed. The associated well-posed problem (the minimization of the "relaxed" energy) cannot be formulated without considering surface phases on the wall. This notion of surface phases is connected with the notion of wetting (or dewetting) films. Rigorous proofs will not be given here: interested readers can refer to [5].

\section{BACK TO THE NO LINE TENSION CASE}

Let us first consider the classical case when no line tension is present. The equilibrium conditions are well known [6]:

- the interface has a constant mean curvature;

- the contact angle $\theta$ is constant along the contact line and is given by the Young's law:

$$
\cos (\theta)=\frac{\gamma_{A \Omega}-\gamma_{B \Omega}}{\gamma_{A B}}
$$

Clearly, when $\left|\gamma_{A \Omega}-\gamma_{B \Omega}\right|>\gamma_{A B}$, the Young's law cannot be satisfied. Different attitudes are possible in this situation:

- one can first consider that the wetting inequality $\gamma_{A B} \geq$ $\left|\gamma_{A \Omega}-\gamma_{B \Omega}\right|$ holds in every physical case;

- one can assume that there is no contact between the phase

$A$ and the wall if this inequality is not satisfied.

Both attitudes cannot be entirely correct: many cases have been described in which the wetting inequality is not satisfied and, when the volume of the phase $A$ is sufficiently large, the contact between $A$ and the wall cannot be avoided.

Assume, for instance, that $\gamma_{A \Omega}>\gamma_{A B}+\gamma_{B \Omega}$. In that case the minimization of the energy $E$ is an ill-posed problem. Indeed, let us consider a minimizing sequence (one can imagine either a slow motion of the phases toward the equilibrium state or a numerical descent method for searching the minimum of the functional). In some geometric cases as the one represented in Figure 2, the limit of the minimizing sequence may not be a minimizer.

From a microscopic (infinitesimal) point of view $S_{A \Omega}$ is empty and the second attitude is correct.

From a macroscopic point of view, the equilibrium configuration is the limit of the minimizing sequence and $S_{A \Omega}$ is not empty: $A$ does not minimize the original energy $E$ but a “relaxed" energy $\tilde{E}$ given by:

$$
\tilde{E}(A):=\gamma_{A B}\left|S_{A B}\right|+\left(\gamma_{A B}+\gamma_{B \Omega}\right)\left|S_{A \Omega}\right|+\gamma_{B \Omega}\left|S_{B \Omega}\right|
$$

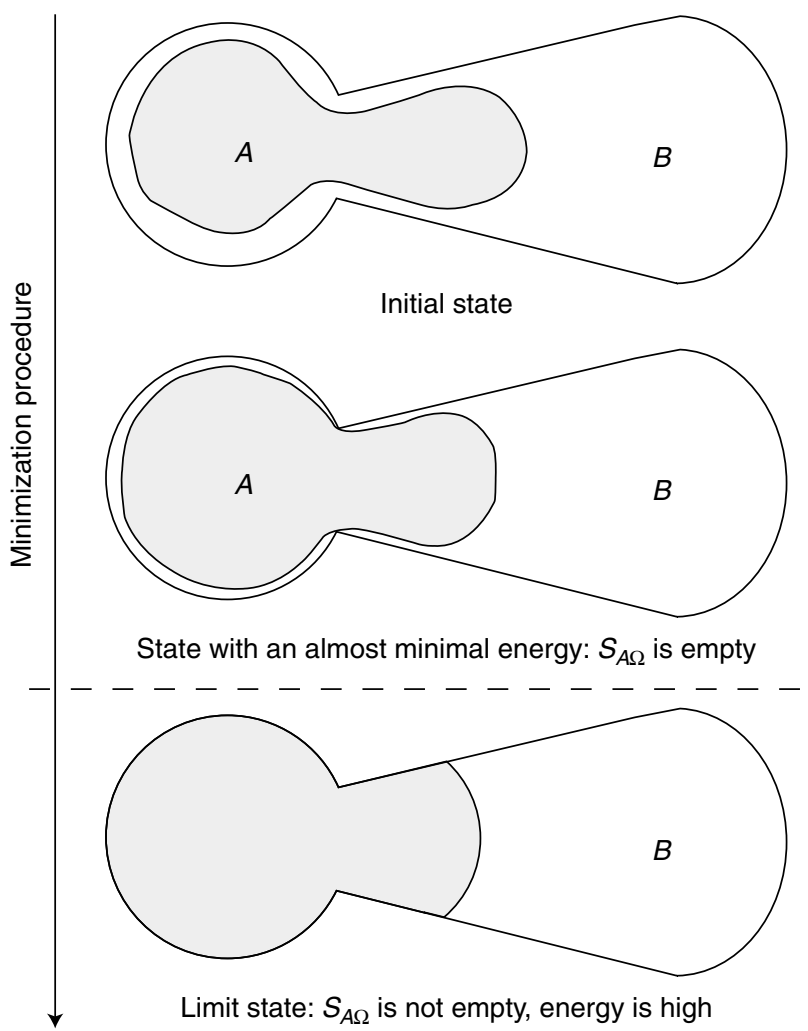

Figure 2

A minimizing sequence.

Then the energy $\tilde{E}$ one has to consider from a macroscopic point of view satisfies the wetting inequality and the first attitude is correct.

The difference between the original energy $E$ and the relaxed one $\tilde{E}$ takes into account the existence of a microscopic (infinitesimal) film of phase $B$ between phase $A$ and the wall. Of course, extra physical arguments may bound the thinness of this film and modify its energy (which is simply here the sum of the energies $\left.\gamma_{A B}+\gamma_{B \Omega}\right)$. Such arguments are not necessary at this point and do not change fundamentally our conclusions.

The remarkable fact we much emphasize is that the relaxed energy $\tilde{E}$ has the same form as the original one $E$. Owing to this "miracle" one can ignore in this model the presence of films along the wall by considering only, from the very beginning, energies which satisfy the wetting condition. The relaxation of the model when line tension is present is not so simple.

\section{INTUITIVE EQUILIBRIUM CONDITIONS}

The equilibrium conditions can be written in an intuitive way by considering the equilibrium of forces at the contact line 


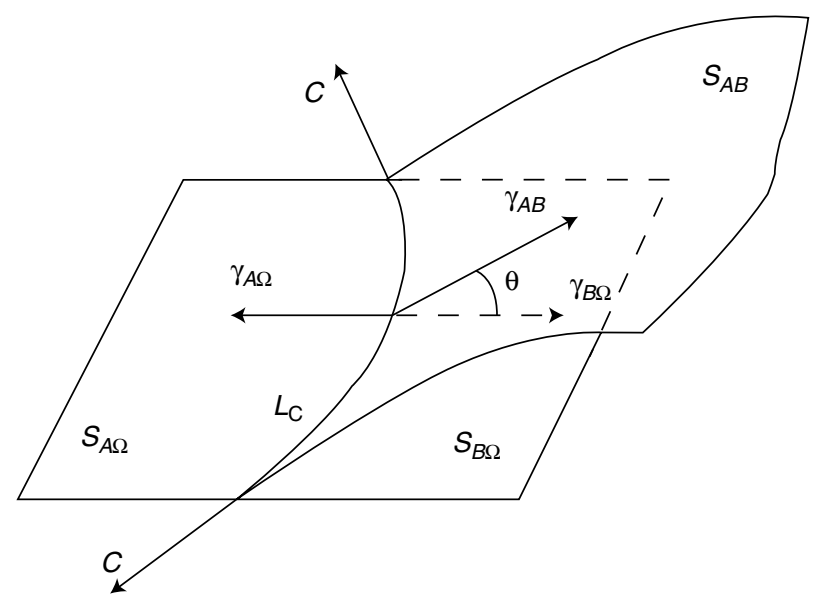

Figure 3

Intuitive equilibrium of the contact line.

(Fig. 3). As previously, the mean curvature of the interface is constant but the Young's law is modified:

$$
\cos (\theta)=\frac{\gamma_{A \Omega}-\gamma_{B \Omega}}{\gamma_{A B}}-\frac{c}{\gamma_{A B}} K
$$

where $K$ denotes the geodesic curvature of the contact line on the wall.

The existence of a contact angle $\theta$ satisfying the Young's law needs the inequality $\gamma_{A B} \geq\left|\gamma_{A \Omega}-\gamma_{B \Omega}-c K\right|$. This condition cannot replace the classical wetting condition as it depends now on the solution (through the curvature $K$ of the contact line). Is there any condition which assures that the equilibrium problem is well-posed?

\section{RELAXED FORMULATION}

Let us compute the relaxed energy by considering again a minimizing sequence (Fig. 4).

The point is that the limits (denoted $A^{\prime}, B^{\prime}$ and $L_{A^{\prime} B^{\prime}}$ ) of $S_{A \Omega}, S_{B \Omega}$ and of the contact line $L_{C}$ do not coincide with the apparent contact surfaces $S_{A \Omega}, S_{B \Omega}$ and contact line $L_{C}$. The surfaces $A^{\prime}$ and $B^{\prime}$ can be considered as surface phases on the wall, the line $L_{A^{\prime} B^{\prime}}$, dividing these phases. The energy of the limit configuration depends on the position of the volume phase $A$ and of the surface phase $A^{\prime}$ (indeed every quantity can be expressed in terms of $A$ and $A^{\prime}$ ):

$$
\begin{aligned}
\tilde{\phi}\left(A, A^{\prime}\right): & =\gamma_{A B}\left|S_{A B}\right|+\gamma_{A A^{\prime}}\left|S_{A A^{\prime}}\right|+\gamma_{B B^{\prime}}, S_{B B^{\prime}}, \mid \\
& +\gamma_{A B},\left|S_{A B},\right|+\gamma_{B A^{\prime}},\left|S_{B A^{\prime}}\right|+c\left|L_{A^{\prime} B^{\prime}},\right|
\end{aligned}
$$

where $S_{A A^{\prime}}$ (respectively $S_{B B^{\prime}}, S_{A B^{\prime}}, S_{B A^{\prime}}$ ) denotes the contact surface between the volume phase $A$ (resp. $B, A, B)$ and the

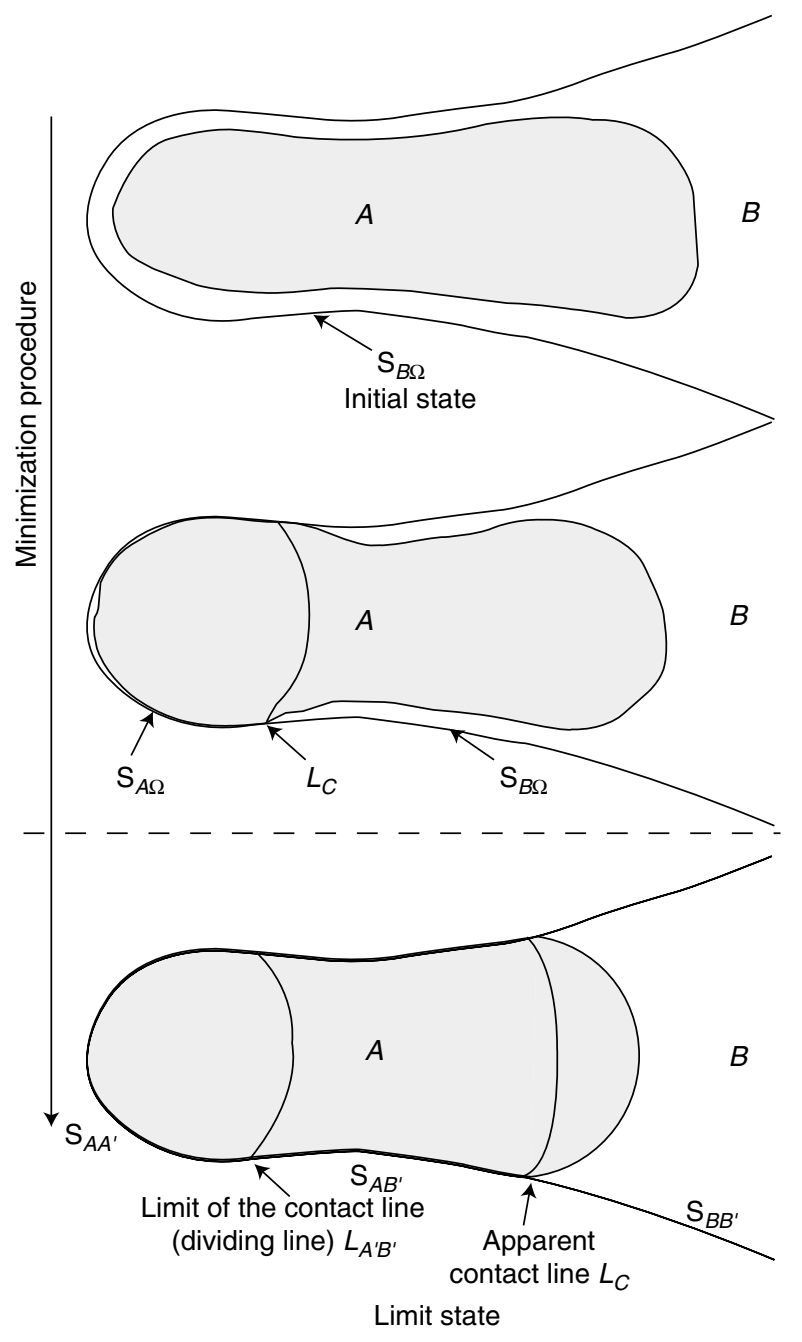

Figure 4

Minimization with line tension.

surface phase $A^{\prime}$ (resp. $\left.B^{\prime}, B^{\prime}, A^{\prime}\right)$. Here the energies are simply given by:

$$
\begin{gathered}
\gamma_{A A^{\prime}}=\gamma_{A \Omega} \\
\gamma_{B B^{\prime}}=\gamma_{B \Omega} \\
\gamma_{A B^{\prime}}=\gamma_{A B}+\gamma_{B \Omega} \\
\gamma_{B A^{\prime}}=\gamma_{A B}+\gamma_{A \Omega}
\end{gathered}
$$

but, as previously, extra physical arguments can modify the value of these energies.

Theoretically, the "relaxed" energy $\tilde{F}$ should be expressed in terms of $A$ only:

$$
\tilde{F}(A)=\inf _{A^{\prime}}, \tilde{\phi}\left(A, A^{\prime}\right)
$$

but this formulation is useless: $\tilde{F}$ is non-local and nonexplicit. On the other hand, privileging the volume phase in 
the formulation of the energy is now somehow arbitrary. The surface phase plays a symmetric role and even, from an experimental point of view, may be the most accessible quantity.

Thus the only way to study capillary equilibrium with line tension is to consider the energy $\tilde{\phi}$ : surface phases (wetting films) cannot be ignored.

\section{ASSOCIATED EQUILIBRIUM CONDITIONS}

Let us write the equilibrium conditions for the energy $\tilde{\phi}$ in the general case. Note that the contact line and the dividing lines can partially coincide (see Figure 5 where a drop of phase $A$ is lying on a non-flat surface and submitted to some extra external force like gravity). The equilibrium conditions depend on the different possible situations (corresponding to the points $M, N, P, Q, R$ in Figure 5).

Let us define the dimensionless parameters $\theta_{1}, \theta_{2}, \tau$ and the characteristic length $\lambda$ as follows:

$$
\begin{gathered}
\cos \left(\theta_{1}\right)=\frac{\gamma_{A A^{\prime}}-\gamma_{B A^{\prime}}}{\gamma_{A B}} \\
\cos \left(\theta_{2}\right)=\frac{\gamma_{A B^{\prime}}-\gamma_{B B^{\prime}}}{\gamma_{A B}} \\
\tau=\frac{\gamma_{B B^{\prime}}+\gamma_{A B^{\prime}}-\gamma_{A A^{\prime}}-\gamma_{B A^{\prime}}}{\gamma_{A B}} \\
\lambda=\frac{c}{\gamma_{A B}}
\end{gathered}
$$

and assume that $\theta_{1} \geq \theta_{2}$, the other case being similar.

Where the two lines coincide (at point $M$ ) we have:

and:

$$
\theta \in\left[\theta_{1}, \theta_{2}\right]
$$

$$
-2 \lambda K=\tau-\cos \left(\theta_{1}\right)-\cos \left(\theta_{2}\right)+2 \cos (\theta)
$$

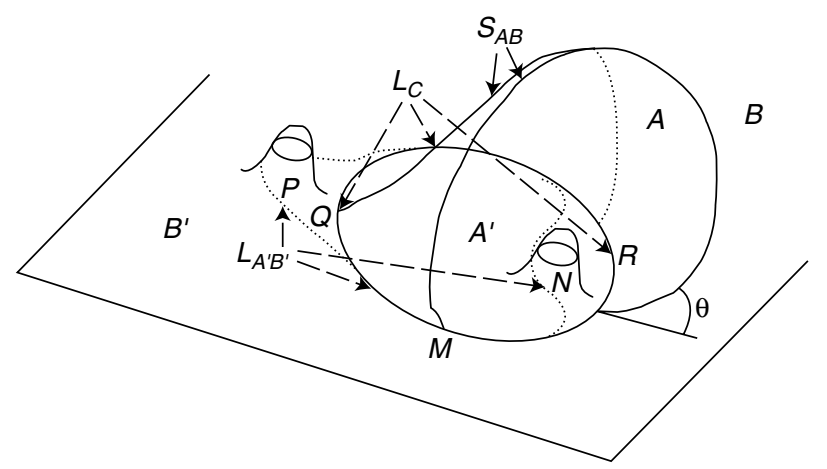

Figure 5

A possible equilibrium state with line tension.
Otherwise:

- on the dividing line $L_{A^{\prime} B^{\prime}}$ :

- in $A$ (at point $N$ ): $-2 \lambda K=\tau-\cos \left(\theta_{1}\right)+\cos \left(\theta_{2}\right)$

- in $B$ (at point $P$ ): $-2 \lambda K=\tau+\cos \left(\theta_{1}\right)-\cos \left(\theta_{2}\right)$

- on the contact line $L_{C}$ :

- in $A^{\prime}$ (at point $Q$ ): $\theta=\theta_{1}$

- in $B^{\prime}$ (at point $R$ ): $\theta=\theta_{2}$

\section{CONSEQUENCES UPON EQUILIBRIUM}

Consequences of these conditions upon equilibrium are straightforward but the possibility for the dividing line and the contact line to separate may lead to hysteresis and instability [7].

For sake of simplicity, assume that $\gamma_{A A^{\prime}}=\gamma_{B B},=0$ and $\gamma_{A B},=\gamma_{B A^{\prime}}=\gamma_{A B}$ and consider a small drop of phase $A$ growing in a capillary tube of radius $r$ (Fig. 6). There are two
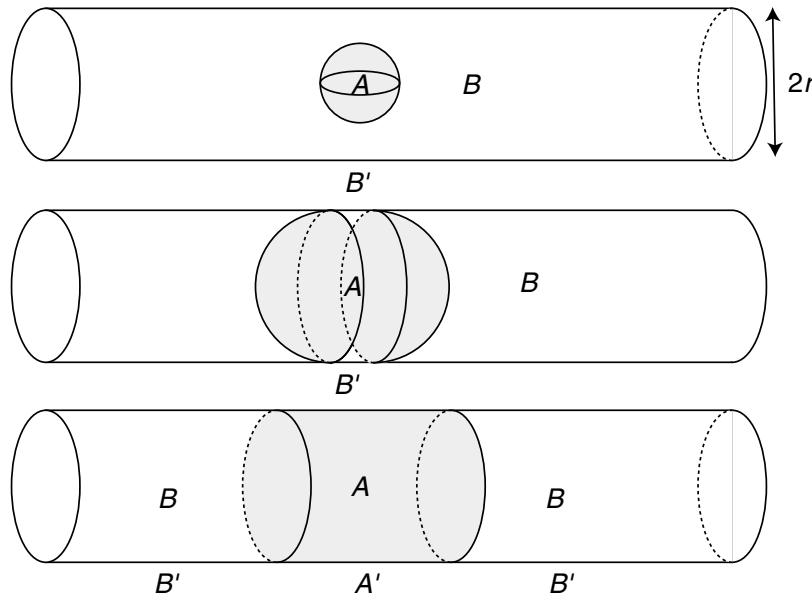

Figure 6

A drop growing in a capillary tube.

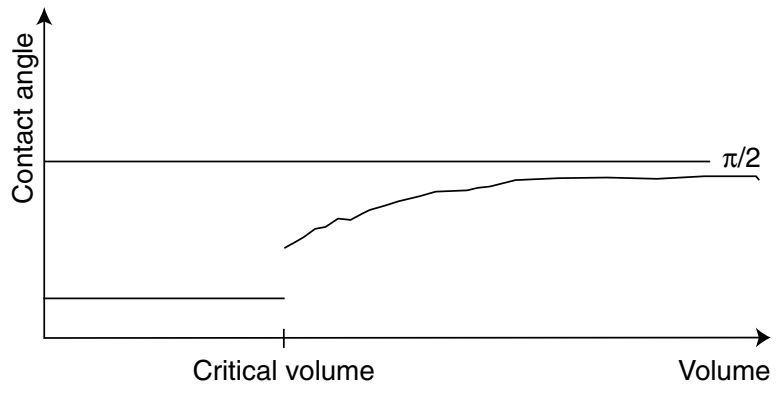

Figure 7

Contact angle of a drop lying on a plane substrate. 
critical volumes for the drop, $V_{1}=4 \pi r^{3} / 3$ and $V_{2}=\pi r^{2}$ $(r / 3+2 \lambda)$ : when $|A|<V_{1}, A$ is a sphere and $A^{\prime}$ is empty; when $V_{1}<|A|<V_{2}, A$ is the union of two half-spheres and a cylinder, $A$ ' is still empty; when $|A|>V_{2}, A$ is a cylinder and $A^{\prime}$ coincides with the interface $S_{A \Omega}$.

Note the sudden change of the contact angle (from 0 to $\pi / 2$ ) when the volume reaches the critical volume $V_{2}$. This result can be extended to a drop growing on a plane surface with $\gamma_{A A^{\prime}}=\gamma_{B B^{\prime}}=0$ and $0<\gamma_{A B^{\prime}}=\gamma_{B A^{\prime}},<\gamma_{A B}$. The computation of the critical volume is less simple [7]: Figure 7 shows the contact angle versus the volume of the drop.

\section{CONCLUSION}

The notion of surface phases (films) is crucial when taking into account line tension. The macroscopic energy $\tilde{\phi}$ must be written in terms of both volume and surface phases which play a symmetrical role. Searching equilibrium configurations with this formulation is straightforward: an instability can occur which is due to the possibility for the dividing line (the line where energy is concentrated) to coincide for a moment with the contact line and then to jump to a lower-energy position.

The capillary equilibrium with line tension appears to be a coupled problem of volume and surface phase transition. This makes clear the impossibility to deal with negative line tension (in the same way as for negative surface tension in the classical model of capillarity): with negative line tension, the minimization problem would be again ill-posed and any configuration would be fundamentally unstable. Negative line tension, invoked for instance in $[1,3,4]$, needs a more sophisticated model: it cannot be taken into account without adding some extra stabilizing energy.

\section{REFERENCES}

1 Rowlinson, J.S. and Widom, B. (1984) Molecular Theory of Capillarity, Clarendon Press, Oxford.

2 Widom, B. and Widom, H. (1991) Model for Line Tension in Three-Phase Equilibrium. Physica A, 173, 72-110.

3 Dussaud, A.D. and Vignes-Adler, M. (1997) Line Tension Effect on Alkane Droplets near the Wetting Transition. Mat. Res. Soc. Symp. Proc., 464, 287-293.

4 Dussaud, A.D. and Vignes-Adler, M. (1997) Wetting Transition of $n$-alkanes on Concentrated Aqueous Salt Solutions. Line Tension Effect. Langmuir, 13, 581-589.

5 Alberti, G., Bouchitté, G. and Seppecher, P. (1998) Phase Transition with Line Tension Effect. Arch. Rational Mech. Anal., 144, 1-46.

6 Finn, R. (1986) Equilibrium Capillary Surfaces, Springer, New York.

7 Widom, B. (1995) Line Tension and the Shape of a Sessile Drop. J. Phys. Chem., 99, 2803-2806.

Final manuscript received in September 2000 